\title{
The estrogen-related receptor $\gamma$ modulator, GSK5182, inhibits osteoclast differentiation and accelerates osteoclast apoptosis
}

\author{
Hyun-Ju Kim ${ }^{*}$, Hye-Jin Yoon, Dong-Kyo Lee, Xian Jin, Xiangguo Che E Je-Yong Choi \\ Department of Biochemistry and Cell Biology, Cell and Matrix Research Institute, Korea Mouse Phenotyping Center, KNU Convergence \\ Educational Program of Biomedical Sciences for Creative Future Talents, School of Medicine, Kyungpook National University, Daegu \\ 41944, Korea
}

Estrogen-related receptor $\gamma($ ERR $\gamma)$, a member of the orphan nuclear receptor family, is a key mediator in cellular metabolic processes and energy homeostasis. Therefore, ERR $\gamma$ has become an attractive target for treating diverse metabolic disorders. We recently reported that $E R R \gamma$ acts as a negative regulator of osteoclastogenesis induced by receptor activator of nuclear factor- $k B$ ligand (RANKL). In the present study, we explored the effects of an ERR $\gamma$-specific modulator, GSK5182, on ERR $\gamma$-regulated osteoclast differentiation and survival. Interestingly, GSK5182 increased ERR $\gamma$ protein levels much as does GSK4716, which is an ERR $\gamma$ agonist. GSK5182 inhibited osteoclast generation from bone-marrow-derived macrophages without affecting cytotoxicity. GSK5182 also attenuated RANKL-mediated expression of cFos and nuclear factor of activated T-cells cytoplasmic 1 (NFATc1), pivotal transcription factors for osteoclastogenesis. Arrested osteoclast differentiation was associated with reduced RANK expression, but not with the M-CSF receptor, c-Fms. GSK5182 strongly blocked the phosphorylation of IKB $\alpha$, c-Jun $\mathrm{N}$-terminal kinase, and extracellular signal-regulated kinase in response to RANKL. GSK5182 also suppressed NF-KB promoter activity in a dose-dependent manner. In addition to osteoclastogenesis, GSK5182 accelerated osteoclast apoptosis by caspase-3 activation. Together, these results suggest that GSK5182, a synthetic ERR $\gamma$ modulator, may have potential in treating disorders related to bone resorption. [BMB Reports 2021; 54(5): 266-271]

\section{INTRODUCTION}

Bone is a very active tissue that experiences continual remodeling by dynamic communication between bone-degrading osteo-

*Corresponding authors. Hyun-Ju Kim, Tel: +82-53-420-4828; Fax: +82-53-422-1466; E-mail: biohjk@knu.ac.kr; Je-Yong Choi, Tel: +8253-420-4823; Fax: +82-53-422-1466; E-mail: jechoi@knu.ac.kr

https://doi.org/10.5483/BMBRep.2021.54.5.243

Received 4 November 2020, Revised 18 November 2020, Accepted 14 December 2020

Keywords: Apoptosis, ERR $\gamma$, GSK5182, NFATc1, Osteoclast clasts and bone-forming osteoblasts. The balance between their cellular functions is crucial for maintaining adult bone mass and integrity. Abnormally elevated bone resorption by osteoclasts breaks this balance and often causes pathologic bone diseases, such as osteoporosis and inflammatory arthritis (1-3). Therefore, regulating osteoclast differentiation, function, and survival is a promising strategy for treating erosive bone disorders.

Osteoclasts are giant polykaryons generated from hematopoietic progenitor cells under the control of macrophage colonystimulating factor (M-CSF) and receptor activator of nuclear factor (NF)-кB ligand (RANKL) (4-7). RANKL binding to its receptor, RANK, triggers osteoclastogenesis by activating downstream signaling pathways, including NF- $\kappa B$ and mitogen-activated protein kinases (MAPKs), c-Jun N-terminal kinase (JNK), extracellular signal-regulated kinase (ERK), and p38 $(8,9)$. In addition, RANKL mediates c-Fos induction and activates calcium signaling by cooperating with immunoglobulin-like receptors. These signaling cascades ultimately induce nuclear factor of activated T-cells cytoplasmic 1 (NFATc1) expression, which upregulates expression of osteoclast marker genes $(10,11)$.

Estrogen-related receptors (ERRs), termed ERR $\alpha, E R R \beta$, and $E R R \gamma$, are orphan receptors that belong to the nuclear receptor superfamily (12-15). ERR $\alpha$ and ERR $\gamma$ play a role in regulating metabolic gene and energy metabolism, whereas ERR $\beta$ maintains embryonic stem-cell pluripotency. Although the endogenous ERR ligands have not been found to date, synthetic compounds that regulate their activity and expression have been developed. Among them, a small synthetic molecule, GSK5182 (4-[(Z)-1-[42-dimethylaminoethyloxy)phenyl]-hydroxy-2-phenylpent-1-eny I]phenol, a 4-hydroxy tamoxifen analog), has been identified as an ERR $\gamma$-specific inverse agonist that does not bind to other nuclear receptors (16). GSK5182 has an anti-diabetic effect by suppressing hepatic gluconeogenesis in a type 2 diabetic mouse model (17). GSK5182 also prevents vascular calcification by downregulating expression of osteogenic marker genes (18). In addition, GSK5182 reduces osteoarthritis (OA) in an experimental animal OA model (19). A recent study also showed that GSK 5182 has anti-tumor activity in human breast cancer, where reactive oxygen species (ROS) play an important role (20). These findings suggest that GSK5182 may help treat various types of human diseases.

ISSN: 1976-670X (electronic edition)

Copyright (C) 2021 by the The Korean Society for Biochemistry and Molecular Biology

(c) This is an open-access article distributed under the terms of the Creative Commons Attribution Non-Commercial License (http://creativecommons.org/licenses/by-nc/4.0) which permits unrestricted non-commercial use, distribution, and reproduction in any medium, provided the original work is properly cited. 
The role of ERR $\gamma$ in bone metabolism has been reported, with most studies demonstrating its function in osteoblast and cartilage formation $(19,21-23)$. Recently, we reported the role of ERR in osteoclast differentiation (24). We showed that ERR $\gamma$ expression is downregulated during the osteoclastogenesis and that ERR $\gamma$ plays a key role in osteoclastogenesis modulation. In the current study, we evaluated the in vitro pharmacological effect on ERR $\gamma$ regulation in osteoclast differentiation and survival by using GSK5182. We show that GSK5182 inhibits osteoclastogenesis by blocking RANKL signaling molecules, demonstrating that this compound may help treat osteoclast-related disorders. Interestingly, GSK5182 increased ERR $\gamma$ protein levels, and ERR $\gamma$ mediated NF- $\mathrm{KB}$ promoter suppression was further accelerated by GSK5182. Therefore, in this context, GSK5182 acts as an agonist of ERR $\gamma$.

\section{RESULTS}

The ERR $\gamma$ modulator, GSK5182, increases ERR $\gamma$ content We first investigated the effect of the synthetic ERR $\gamma$ modulators, GSK5182 and GSK4716, on ERR $\gamma$ protein expression. We isolated and cultured bone-marrow-derived macrophages (BMMs) with two different concentrations of ERR $\gamma$ modulators. Immunoblotting analysis using an ERR $\gamma$-specific antibody showed that GSK5182 treatment significantly increased ERR $\gamma$ protein levels in a dose-dependent manner (Fig. 1A). We also observed that GSK4716, a known ERR $\gamma$ agonist compound, elevated ERR $\gamma$ protein levels at both concentrations (Fig. 1B). We then examined whether GSK5182 affects ERR $\gamma$ expression at the RNA level. As determined by real-time PCR, GSK5182 dose-dependently increased mRNA expression of ERR $\gamma$ (Fig. 1C). These results demonstrate that GSK5182 regulates ERR $\gamma$ expression at the transcriptional level, but the precise mechanism of this modulation remains to be further investigated.

GSK5182 blocks osteoclast formation induced by RANKL To explore GSK5182's effect on osteoclast formation, we cultured BMMs in the presence of RANKL, M-CSF, and various GSK 5182 doses or vehicles. Compared with the vehicle-treated control, GSK5182 exposure inhibited tartrate-resistant acid phosphatase (TRAP)-expressing multinucleated osteoclast generation from BMMs in a dose-dependent manner (Fig. 1D, E). GSK5182 completely blocked osteoclast formation at $10 \mu \mathrm{M}$ (Fig. 1E). Consistent with a decrease in osteoclast number, TRAP activity from culture supernatants was also dose-dependently reduced by GSK5182 (Fig. 1F). Employing an MTS assay, we also found that the GSK5182 concentrations used in this study did not influence BMM cytotoxicity or proliferation (Fig. 1G).

\section{GSK5182 attenuates osteoclastogenic marker induction} Because GSK5182 inhibited osteoclast formation, we next evaluated the GSK5182 effect on expression of osteoclastogenesisassociated marker genes. We cultured BMMs with RANKL and $\mathrm{M}$-CSF in the absence or presence of GSK5182 $(10 \mu \mathrm{M})$. RANKL

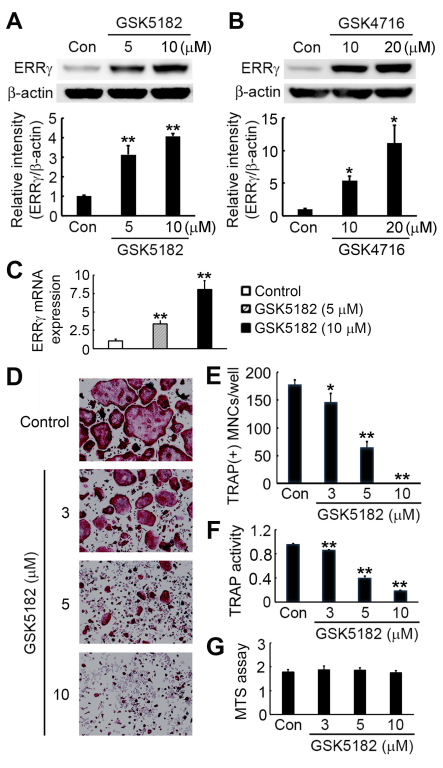

Fig. 1. GSK5182 inhibits RANKL-induced osteoclast formation. We cultured BMMs with the indicated concentrations of ERR $\gamma$ modulators GSK5182 (A) or GSK4716 (B) for $24 \mathrm{~h}$. We used immunoblotting to detect the ERRy protein contents. Graphs represent the results of quantitative analysis of ERR $\gamma$ protein levels from three independent experiments. (C) We did real-time PCR to assess ERR $\gamma$ mRNA levels. (D-F) BMMs were cultured at the indicated GSK5182 concentrations in the presence of RANKL $(20 \mathrm{ng} / \mathrm{ml})$ and M-CSF $(20 \mathrm{ng} / \mathrm{ml})$ for four days. (D) The cells were fixed and stained for TRAP. (E) TRAP-positive multinucleated cells (MNCs) were counted. (F) TRAP activity in the culture medium generated by cells in (D) was quantified at $405 \mathrm{~nm}$. (G) We cultured BMMs with the indicated concentrations of GSK5182 in the presence of M-CSF $(30 \mathrm{ng} / \mathrm{ml})$ for three days. We evaluated cell viability by MTS assay. The data presented in (A-C, E, and F) are expressed as the mean $\pm \mathrm{SD}$. $* P<0.05, * * P<0.001$ vs. the vehicle-treated control (Con).

stimulation in control BMMs increased c-Fos and NFATc1 mRNA levels, two key proteins involved in RANKL-mediated osteoclast differentiation. However, GSK5182 significantly attenuated the mRNA induction of these two transcription factors (Fig. 2A). These suppressive effects were further revealed by Western blot analysis. GSK5182 strongly reduced c-Fos and NFATc1 protein expression in a time- and dose-dependent manner (Fig. 2B, C). Reflecting the decrease in NFATc1 protein level, the expression of NFATc1 target genes, TRAP and cathepsin K (CTSK), was also attenuated in response to GSK5182 (Fig. 2A, B). We also found that GSK5182 suppressed the mRNA expression of the RANKL receptor, RANK, but not of the M-CSF receptor, c-Fms (Fig. 2A). We obtained similar results in the Western blot, showing that RANK protein levels were significantly reduced by GSK5182 treatment (Fig. 2D).

\section{GSK5182 inhibits RANKL-stimulated NF-KB, JNK, and ERK activation}

To further understand the mechanism for GSK5182's inhibi- 

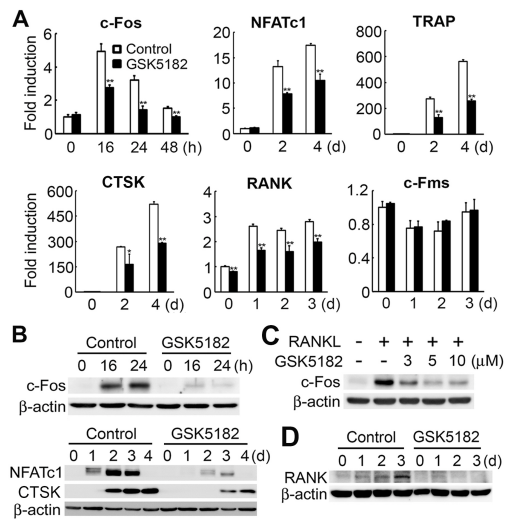

Fig. 2. GSK5182 suppresses osteoclastogenic marker expression. (A and B) We cultured BMMs with RANKL and M-CSF in the absence or presence of GSK5182 $(10 \mu \mathrm{M})$ for the indicated times. We did real-time PCR (A) or immunoblotting (B) to assess the expression of the indicated genes. ${ }^{*} \mathrm{P}<0.05,{ }^{*} * \mathrm{P}<0.001$ vs. control. (C) We cultured BMMs with M-CSF in the absence $(-)$ or presence $(+)$ of RANKL with various GSK5182 concentrations for $24 \mathrm{~h}$. Whole-cell lysates were subjected to immunoblotting to detect c-Fos. (D) RANK protein expression in cells treated with GSK5182 $(10 \mu \mathrm{M})$ on the indicated days.

tion of RANKL-induced osteoclastogenesis, we examined the GSK5182 effect on RANKL signaling cascades. We pretreated BMMs with GSK5182 or vehicle and assessed them for MAPK activation in response to RANKL. As expected, RANKL exposure in control cells activated MAPKs, including JNK, ERK, and p38 (Fig. 3A). In contrast, BMMs pretreated with GSK5182 abrogated RANKL-stimulated JNK and ERK phosphorylation (Fig. 3A). However, p38 activation by RANKL was not altered in the presence of GSK5182 (Fig. 3A).

Another RANKL signaling cascade important for osteoclastogenesis is the NF- $\kappa B$ signaling pathway. RANKL stimulation elevated $I \kappa B \alpha$ phosphorylation levels in vehicle-treated control BMMs, whereas GSK5182 strongly inhibited RANKL-induced $I_{\kappa} B \alpha$ phosphorylation (Fig. 3B). Accordingly, I $\mathrm{KB} \alpha$ protein amounts were increased in GSK5182-exposed cells at both concentrations (Fig. 3C). RANKL also triggers the induction of the NF-KB transcriptional activity. Hence, we next assessed the effect of GSK5182 on RANKL-mediated NF- $\mathrm{KB}$ promoter activity in RAW 264.7 macrophages. RANKL exposure increased NF-KB luciferase activity, whereas GSK5182 led to a dose-dependent reduction in NF-KB activity following RANKL stimulation (Fig. 3D). As previously reported (24), the induction of NF-kB promoter activity was significantly attenuated by ERR $\gamma$ overexpression (Fig. 3E). Notably, GSK5182 treatment accelerated the ERR $\gamma$-reducing effect on the NF-kB promoter in a dose-dependent manner (Fig. 3E). These results reveal that GSK5182 inhibits the transactivation of NF-KB, much as ERR $\gamma$ does, indicating that GSK5182 can act on ERR $\gamma$ in an agonistic manner.

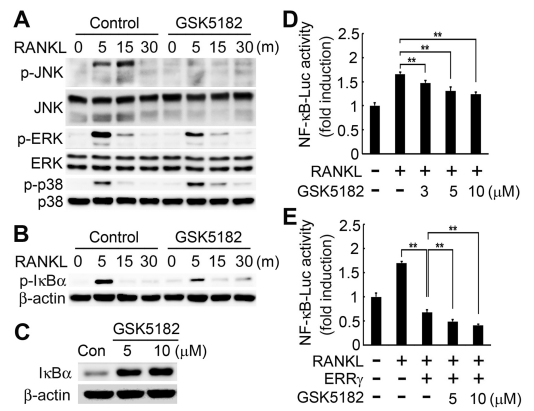

Fig. 3. GSK5182 blocks RANKL-stimulated NF- $\mathrm{KB}, \mathrm{JNK}$, and ERK activation. (A and B) BMMs were serum-starved for $6 \mathrm{~h}$, pretreated with GSK5182 $(10 \mu \mathrm{M})$ or vehicle (control) for $1 \mathrm{~h}$, and then stimulated with RANKL (30 ng/ml). (A) We assayed total protein extracts by immunoblotting to detect phosphorylated and total MAPK (JNK, ERK, p38) proteins. (B) Cell lysates were immunoblotted with a phospho$I_{\kappa} \mathrm{B} \alpha$ antibody. (C) $I_{\kappa} \mathrm{B} \alpha$ protein levels in GSK5182-treated cells. (D, E) RAW264.7 cells were transfected with NF- $\kappa B$ luciferase reporter alone (D) or co-transfected with NF-kB luciferase together with the ERR $\gamma$ expression plasmid (E). After transfection, we treated cells with the indicated concentrations of GSK5182 or vehicle for $24 \mathrm{~h}$ and then stimulated them with RANKL $(100 \mathrm{ng} / \mathrm{ml})$ for $24 \mathrm{~h}$. We measured $\mathrm{NF}-\kappa \mathrm{B}$ luciferase activity using a dual-luciferase reporter assay system. $* * \mathrm{P}<0.001$.

\section{GSK5182 accelerates osteoclast apoptosis}

Because osteoclastic bone resorption is also governed by the cell-survival rate, we next investigated the GSK5182 effect on osteoclast survival. We incubated mature osteoclasts with or without GSK5182 for $24 \mathrm{~h}$ and then stained them for TRAP (Fig. 4A). GSK5182 strongly decreased the survival rate of TRAPpositive osteoclasts (Fig. 4B). To examine if the reduced cell-survival rate was accompanied by apoptosis in GSK5182-treated cells, we carried out an apoptotic DNA fragmentation assay by ELISA. GSK5182 markedly increased apoptosis of mature osteoclasts (Fig. 4C). In agreement with its pro-apoptotic effect, GSK5182 accelerated both caspase-3 and caspase-9 activity (Fig. 4D).

\section{DISCUSSION}

Because ERR $\gamma$ is implicated in the pathogenesis of various metabolic events, regulating ERR $\gamma$ using small synthetic compounds provides a useful clue for a novel therapeutic strategy. ERR $\gamma$ is an orphan nuclear receptor because of the absence of its natural ligand. In this regard, a small synthetic compound, GSK5182, has attracted attention as a highly specific ERR $\gamma$ modulator that does not associate with other nuclear receptors. To date, several studies have highlighted the therapeutic GSK5182-mediated effects on regulating ERR $\gamma$ in a variety of metabolic diseases (17-20, 2527). Along with these previous studies, our recent study also demonstrates that regulating ERR $\gamma$ may be a way to treat bone disorders in an excessive bone-resorption pathological state. Employing loss- and gain-of-function systems, we reported that ERR $\gamma$ negatively controls RANKL-induced osteoclast differentiation (24). In the current study, we show that an ERR modu- 


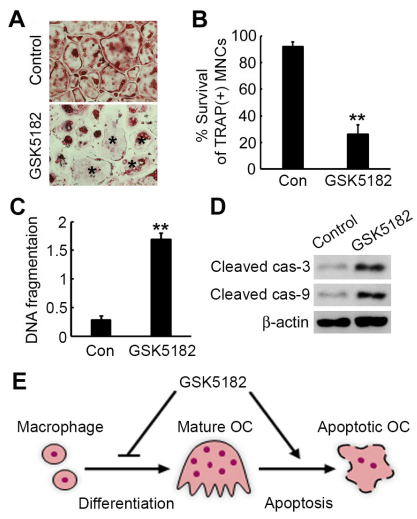

Fig. 4. GSK5182 promotes osteoclast apoptosis. We incubated mature osteoclasts with GSK5182 $(10 \mu \mathrm{M})$ or vehicle for $24 \mathrm{~h}$. (A) The cells were fixed and subjected to TRAP staining. Apoptotic osteoclasts are marked by asterisks. (B) We measured osteoclast survival as the TRAPpositive MNC percentage. ${ }^{* * P}<0.001$. (C) We measured the osteoclast apoptosis rate by ELISA. $* * P<0.001$. (D) Total cell lysates were immunoblotted for cleaved caspase (cas)-3 and caspase-9. $\beta$-Actin served as a loading control. (E) A diagram showing the GSK5182 effect on osteoclast differentiation and apoptosis.

lator, GSK5182, inhibits osteoclastogenesis and promotes mature osteoclast apoptosis, suggesting that GSK5182 might work well to treat bone-resorption-associated diseases (Fig. 4E).

In an attempt to explore GSK5182's effect on osteoclast formation, we first examined changes in ERR $\gamma$ protein levels. We found that GSK4716, an ERR $\gamma$ agonist, increased ERR $\gamma$ protein levels. Our recent study demonstrated that both ERR overexpression and GSK4716 treatment inhibited RANKL-mediated osteoclastogenesis (24), indicating that GSK4716 is indeed an ERR $\gamma$ agonist. Interestingly, GSK5182, a compound identified as an ERR $\gamma$ inverse agonist, also dose-dependently increased ERR $\gamma$ protein levels. Furthermore, like ERR $\gamma$ and GSK4716, GSK 5182 blocked osteoclast formation and attenuated osteoclastogenic marker gene expression. These findings suggest that GSK 5182-mediated ERR $\gamma$ protein increase may impair osteoclast differentiation. Therefore, we conclude that GSK5182 functions as an agonist and expression modulator of ERR $\gamma$, which serves as a strong negative regulator of osteoclast differentiation. The GSK5182 behavior is similar to that of the ERR $\alpha$ modulator, XCT790. A previous study showed that XCT790 is an ERR $\alpha$ antagonist that is also an ERR $\alpha$ agonist that suppresses cell proliferation and tumorigenicity (28). Thus, like XCT790, GSK5182 may act in a cell- and/or context-specific manner as a receptor agonist or antagonist.

c-Fos and NFATc1 are critical transcription factors for the RANKL-induced osteoclastogenesis. c-Fos targeted disruption results in severe osteopetrosis because it fails to generate osteoclasts from their precursors $(29,30)$. NFATc1-deficient mouse embryonic stem cells could not form osteoclasts following RANKL stimulation (31). Also, c-Fos is recruited to the NFATc1 promoter after $24 \mathrm{~h}$ of RANKL exposure (32), indicating that
NFATc1 is a c-Fos target gene during the early stages of osteoclastogenesis. In this study, we observed that GSK5182 attenuated c-Fos and NFATc1 mRNA and protein expression. Consequently, GSK5182 suppressed NFATc1 target genes, such as TRAP and cathepsin K. These data reveal that GSK5182 inhibits osteoclast differentiation by suppressing the two key transcription factors, c-Fos and NFATc1.

$\mathrm{NF}-\kappa \mathrm{B}$ activation by RANKL is a critical signaling event for osteoclast differentiation. Deleting the NF- $\mathrm{KB}$ subunits (p50 and $\mathrm{p} 52$ ) leads to severe osteopetrosis because of defective osteoclastogenesis $(33,34)$. NF-KB is also recruited to the NFATc1 promoter in response to RANKL (32). We found that GSK5182 strongly blocked RANKL-stimulated phosphorylation of the NF- $\kappa B$ inhibitor, I $\mathrm{\kappa B} \alpha$. In agreement with this observation, ERR $\gamma$ overexpression and DY131 treatment, another ERR agonist, inhibited I $\mathrm{kB} \alpha$ phosphorylation induced by RANKL (24). Furthermore, GSK5182 attenuated RANKL-mediated NF-кB transcriptional activity. As NF- $\kappa B$ promoter activity was also suppressed by ERR $\gamma$, and this ERR $\gamma$ inhibitory effect was further accelerated by GSK5182, in this circumstance, GSK5182 functions as a receptor agonist. RANKL stimulation also activates the MAPK signaling pathways, including JNK, ERK, and p38. GSK 5182 inhibited JNK and ERK activation by RANKL, whereas this compound did not influence p38 activation. Collectively, our data reveal that blunting the RANKL-stimulated NF-KB, JNK, and ERK signaling pathways contributes to the suppressive effect of GSK5182 on NFATc1 induction.

Apoptosis of mature osteoclasts and/or osteoclast precursor cells is another important factor that governs osteoclast number and bone-resorptive capacity. In addition to its anti-osteoclastogenic effect, GSK5182 increased fully differentiated osteoclast apoptosis. GSK5182 increased mature osteoclast cell death by inducing caspase- 3 and caspase- 9 activation. However, this compound did not affect osteoclast precursor (BMM) cell viability (Fig. 1F).

In conclusion, our data showed that GSK5182 efficiently blocks osteoclast differentiation. The preventive effect of GSK5182 is associated with inhibiting c-Fos, NF- $\mathrm{BB}$, JNK, and ERK signaling that leads to NFATc1 downregulation. Additionally, GSK5182 promotes osteoclast apoptosis by inducing caspase activation. Therefore, our findings suggest that the ERR $\gamma$ modulator, GSK5182, might help treat skeletal diseases accompanied by excessive bone erosion, such as osteoporosis, periodontitis, and rheumatoid arthritis.

\section{MATERIALS AND METHODS}

\section{Reagents and antibodies}

GSK5182 was synthesized as described previously (16). We purchased:

- Recombinant murine RANKL and human M-CSF from R\&D Systems (Minneapolis, MN, USA).

- Primary antibodies specific for $1 \kappa B \alpha, J N K$, p38, ERK, phospho-IKB $\alpha$, phospho-JNK, phospho-p38, and phospho-ERK 
from Cell Signaling Technology (Beverly, MA, USA).

- ERR $\gamma$ from R\&D Systems.

- NFATc1 from BD Pharmingen (San Diego, CA, USA).

- c-Fos from Santa Cruz Biotechnology (Santa Cruz, CA, USA).

- RANK from Abcam (Cambridge, UK).

\section{Osteoclast differentiation}

We prepared osteoclasts from murine bone-marrow cells as previously described $(35,36)$. Whole bone marrow from C57/ BL6 male mice femurs and tibiae was flushed out with $\alpha$-minimum essential media ( $\alpha$-MEM). After removing the red blood cells, we incubated bone-marrow cells for three days in Petri dishes with $\alpha$-MEM containing 10\% FBS and 10\% CMG 14-12 culture media (37) as a source of M-CSF. We selected the adherent cells as bone-marrow-derived macrophages (BMMs). To induce osteoclasts, we cultured precursor cells (BMMs) in $\alpha$ MEM with RANKL $(20 \mathrm{ng} / \mathrm{ml})$ and M-CSF (20 ng/ml) for four or five days. Cells were fixed in $4 \%$ paraformaldehyde and stained with TRAP.

\section{Cell viability assay}

We seeded BMMs at $5 \times 10^{3}$ cells per well in 96-well plates and cultured them with different GSK5182 doses in the presence of M-CSF $(30 \mathrm{ng} / \mathrm{ml})$. After three days, the cells were incubated with $20 \mu \mathrm{l}$ per well of CellTiter 96 AQueous One Solution Reagent containing MTS (Promega, Madison, WI, USA) at $37^{\circ} \mathrm{C}$ for $4 \mathrm{~h}$. We quantified cell viability by measuring absorbance at $490 \mathrm{~nm}$.

\section{Real-time PCR}

We did quantitative real-time PCR using SYBR Green dye and an ABI 7500 Real-Time PCR system (Applied Biosystems, Foster City, CA, USA). The following primer sequences were used:

- ERR $\gamma, 5^{\prime}$-GCTAAGTGCCAGAATTCAAACCA-3' and 5'-ATC GAC TCCTATGGATCAGGACTTTT-3';

- c-Fos, 5'-AGGCCCAGTGGCTCAGAGA-3' and 5'-GCTCCCA GTCTGCTGCATAGA-3';

- NFATC1, 5'-ACCACCTTTCCGCAACCA-3' and 5'-TTCCGTTा CCCGTTGCA-3';

- TRAP, 5'-TCCCCAATGCCCCATTC-3' and 5'-CGGTTCTGGC GATCTCTTTG-3';

- Cathepsin K, 5'-GGCTGTGGAGGCGGCTAT-3' and 5'-AG AGTCAATGCCTCCGTTCTG-3';

- RANK, 5'-TCTGCAGCTCTTCCATGACACT-3' and 5'-GAAG AGGAGCAGAACGATGAGACT-3';

- c-Fms, 5'-CCTGAATCTCCCGGAAGCA-3' and 5'-CAAGCTC GGTACAACGGTAGGT-3'.

\section{Western blotting}

We harvested vehicle or compound-treated cells and lysed them in RIPA buffer (Sigma-Aldrich, St. Louis, MO, USA) supplemented with protease-phosphatase inhibitors. We measured the protein concentration using a BCA protein assay kit (Pierce Biotech- nology, Rockford, IL, USA). Equivalent protein amounts were subjected to SDS-PAGE and Western blotting analysis. The immunoreactive proteins were detected using an ECL Plus kit (Amersham Pharmacia Biotech, Piscataway, NJ, USA) and imaged with a LAS4000 luminescent image analyzer (GE Healthcare, Piscataway, NJ, USA).

\section{Luciferase assay}

We cultured RAW264.7 macrophages in Dulbecco's modified Eagle's medium containing $10 \%$ FBS at $1 \times 10^{5}$ cells per well in 48-well plates. On the following day, the cells were transfected with a NF- $\kappa$ B reporter plasmid (250 ng) using FuGENE HD transfection reagent (Promega, Madison, WI, USA). In another assay, we co-transfected RAW264.7 cells with the NF-кB luciferase reporter (250 ng) and an ERR $\gamma$ expression plasmid (250 ng) provided by Dr. Hueng-Sik Choi (Chonnam National University, Gwangju, Korea). After $24 \mathrm{~h}$, we incubated the cells with various GSK5182 concentrations for $24 \mathrm{~h}$ and then stimulated them with RANKL (100 ng/ml) for $24 \mathrm{~h}$. We measured NF-kB luciferase activity using a dual-luciferase reporter assay system (Promega) according to the manufacturer's protocols.

\section{Apoptosis assay}

We cultured mature osteoclasts in the presence of a vehicle or GSK5182 for one day. Osteoclast apoptosis was analyzed using a Cell Death Detection ELISA ${ }^{\text {PLS }}$ kit (Roche, Mannheim, Germany), which quantitatively detects cytoplasmic histone-associated DNA fragments.

\section{Statistics}

We did statistical analysis using Microsoft Excel 2016 (Microsoft, USA). Statistical significance was found by Student's t-test, considering $\mathrm{P}<0.05$ as significant. The results are represented as the mean \pm standard deviation (SD).

\section{ACKNOWLEDGEMENTS}

This work was supported by the National Research Foundation of Korea (NRF) grant funded by the Korean Government (MSIT) (No. 2018R1A2B6001298); the Basic Science Research Program of NRF grant funded by the Ministry of Education, Science, and Technology (NRF-2015R1D1A1A01056666); and the Bio \& Medical Technology Development Program (NRF-2017R1A5A2015391).

\section{CONFLICTS OF INTEREST}

The authors have no conflicting interests.

\section{REFERENCES}

1. Boyle WJ, Simonet WS and Lacey DL (2003) Osteoclast differentiation and activation. Nature 423, 337-342

2. Teitelbaum SL (2000) Bone resorption by osteoclasts. Science 289, 1504-1508

3. Tsukasaki M and Takayanagi H (2019) Osteoimmunology: 
evolving concepts in bone-immune interactions in health and disease. Nat Rev Immunol 19, 626-642

4. Kong YY, Yoshida H, Sarosi I et al (1999) OPGL is a key regulator of osteoclastogenesis, lymphocyte development and lymph-node organogenesis. Nature 397, 315-323

5. Lacey DL, Timms E, Tan HL et al (1998) Osteoprotegerin ligand is a cytokine that regulates osteoclast differentiation and activation. Cell 93, 165-176

6. Wong BR, Rho J, Arron J et al (1997) TRANCE is a novel ligand of the tumor necrosis factor receptor family that activates c-Jun $\mathrm{N}$-terminal kinase in T cells. J Biol Chem 272, 25190-25194

7. Yasuda H, Shima N, Nakagawa N et al (1998) Osteoclast differentiation factor is a ligand for osteoprotegerin/osteoclastogenesis-inhibitory factor and is identical to TRANCE/ RANKL. Proc Natl Acad Sci U S A 95, 3597-3602

8. Lee $\mathrm{ZH}$ and $\mathrm{Kim} \mathrm{HH}$ (2003) Signal transduction by receptor activator of nuclear factor kappa B in osteoclasts. Biochem Biophys Res Commun 305, 211-214

9. Park JH, Lee NK and Lee SY (2017) Current understanding of RANK signaling in osteoclast differentiation and maturation. Mol Cells 40, 706-713

10. Kim JH and Kim N (2014) Regulation of NFATc1 in osteoclast differentiation. J Bone Metab 21, 233-241

11. Nakashima T, Hayashi M and Takayanagi H (2012) New insights into osteoclastogenic signaling mechanisms. Trends Endocrinol Metab 23, 582-590

12. Giguere V (2008) Transcriptional control of energy homeostasis by the estrogen-related receptors. Endocr Rev 29, 677-696

13. Giguere V, Yang N, Segui P and Evans RM (1988) Identification of a new class of steroid hormone receptors. Nature 331, 91-94

14. Hong H, Yang L and Stallcup MR (1999) Hormone-independent transcriptional activation and coactivator binding by novel orphan nuclear receptor ERR3. J Biol Chem 274, 22618-22626

15. Misra J, Kim DK and Choi HS (2017) ERRgamma: a junior orphan with a senior role in metabolism. Trends Endocrinol Metab 28, 261-272

16. Chao EY, Collins JL, Gaillard S et al (2006) Structure-guided synthesis of tamoxifen analogs with improved selectivity for the orphan ERRgamma. Bioorg Med Chem Lett 16, 821-824

17. Kim DK, Gang GT, Ryu D et al (2013) Inverse agonist of nuclear receptor ERRgamma mediates antidiabetic effect through inhibition of hepatic gluconeogenesis. Diabetes 62, 3093-3102

18. Kim JH, Choi YK, Do JY et al (2015) Estrogen-related receptor gamma plays a key role in vascular calcification through the upregulation of BMP2 expression. Arterioscler Thromb Vasc Biol 35, 2384-2390

19. Son YO, Park S, Kwak JS et al (2017) Estrogen-related receptor gamma causes osteoarthritis by upregulating extracellular matrix-degrading enzymes. Nat Commun 8, 2133

20. Vernier M, Dufour CR, McGuirk S et al (2020) Estrogenrelated receptors are targetable ROS sensors. Genes Dev 34, 544-559

21. Cardelli M and Aubin JE (2014) ERRgamma is not required for skeletal development but is a RUNX2-dependent negative regulator of postnatal bone formation in male mice.
PLoS One 9, e109592

22. Cardelli M, Zirngibl RA, Boetto JF et al (2013) Cartilage-specific overexpression of ERRgamma results in Chondrodysplasia and reduced chondrocyte proliferation. PLoS One 8, e81511

23. Jeong BC, Lee YS, Park YY et al (2009) The orphan nuclear receptor estrogen receptor-related receptor gamma negatively regulates BMP2-induced osteoblast differentiation and bone formation. J Biol Chem 284, 14211-14218

24. Kim HJ, Kim BK, Ohk B et al (2019) Estrogen-related receptor gamma negatively regulates osteoclastogenesis and protects against inflammatory bone loss. J Cell Physiol 234, 1659-1670

25. Kim DK, Jeong JH, Lee JM et al (2014) Inverse agonist of estrogen-related receptor gamma controls Salmonella typhimurium infection by modulating host iron homeostasis. Nat Med 20, 419-424

26. Kim DK, Kim JR, Koh M et al (2011) Estrogen-related receptor gamma (ERRgamma) is a novel transcriptional regulator of phosphatidic acid phosphatase, LIPIN1, and inhibits hepatic insulin signaling. J Biol Chem 286, 38035-38042

27. Kim DK, Ryu D, Koh M et al (2012) Orphan nuclear receptor estrogen-related receptor gamma (ERRgamma) is key regulator of hepatic gluconeogenesis. J Biol Chem 287, 2162821639

28. Bianco S, Lanvin O, Tribollet V, Macari C, North S and Vanacker JM (2009) Modulating estrogen receptor-related receptor-alpha activity inhibits cell proliferation. J Biol Chem 284, 23286-23292

29. Johnson RS, Spiegelman BM and Papaioannou V (1992) Pleiotropic effects of a null mutation in the c-fos proto-oncogene. Cell 71, 577-586

30. Wang ZQ, Ovitt C, Grigoriadis AE, Mohle-Steinlein U, Ruther U and Wagner EF (1992) Bone and haematopoietic defects in mice lacking c-fos. Nature 360, 741-745

31. Takayanagi $H$, Kim S, Koga $T$ et al (2002) Induction and activation of the transcription factor NFATc1 (NFAT2) integrate RANKL signaling in terminal differentiation of osteoclasts. Dev Cell 3, 889-901

32. Asagiri M, Sato K, Usami T et al (2005) Autoamplification of NFATc1 expression determines its essential role in bone homeostasis. J Exp Med 202, 1261-1269

33. Franzoso G, Carlson L, Xing L et al (1997) Requirement for NF-kappaB in osteoclast and B-cell development. Genes Dev 11, 3482-3496

34. Iotsova V, Caamano J, Loy J, Yang Y, Lewin A and Bravo R (1997) Osteopetrosis in mice lacking NF-kappaB1 and NF-kappaB2. Nat Med 3, 1285-1289

35. Kim HJ, Lee DK, Jin X, Che X and Choi JY (2020) Oleoylethanolamide exhibits GPR119-dependent inhibition of osteoclast function and GPR119-independent promotion of osteoclast apoptosis. Mol Cells 43, 340-349

36. Jung YK, Han SW, Kim GW, Jeong JH, Kim HJ and Choi JY (2012) DICAM inhibits osteoclast differentiation through attenuation of the integrin alphaVbeta3 pathway. J Bone Miner Res 27, 2024-2034

37. Takeshita S, Kaji K and Kudo A (2000) Identification and characterization of the new osteoclast progenitor with macrophage phenotypes being able to differentiate into mature osteoclasts. J Bone Miner Res 15, 1477-1488 